CIENCIA Y SOCIEDAD

Volumen XV, Número 2

Abril - Junio 1990

LOS BOSQUES NUBLADOS DE LA REPUBLICA DOMINICANA

ELEUTERIO MARTINEZ ${ }^{*}$

\title{
Introducción
}

Los bosques nublados, la más rara formación forestal de las regiones tropicales de la Tierra y presentes en República Dominicana únicamente en zonas cordilleranas muy apartadas, constituyen los ecosistemas más frágiles e improtantes de los biomas de montañas en cualquier parte del mundo, muy especialmente en territorios insulares sin ninguna influencia continental.

Estos tipos de bosques que se presentan generalmente en elevaciones medias de montañas expuestas a los vientos predominantes, constituyen toda una novedad para la investigación científica y un desafío para los instrumentos meteorológicos más modernos y sofisticados; pues, muy pocos estudios existen al respecto en el ámbito internacional y en nuestro país son prácticamente nulos.

La nubosidad persistente cuya humedad se traspasa directamente a las plantas a través del fenómeno climático conocido como "precipitación horizontal"; la composición florística, grandemente diferenciada y ultraespecializada, tipificada por toda una inmensa gama de cpífitas

* Postgrado en Educación Ambiental. Facultad Ciencias y Humanidades. INTEC. 
(bromelias, orquídeas, helechos, musgos, líquenes), y el aporte adicional de agua a las fuentes acuíferas subterráneas y de escorrentía, son eventos naturales que ameritan ser debidamente conocidos, ponderados y evaluados.

Profesionales de las más diversas disciplinas: ecólogos, botánicos, forestales, meteorólogos, geógrafos, climatólogos, zoólogos, hidrólogos y conservacionistas en general han encontrado en los bosques nublados un elemento nuevo dentro del campo de la investigación científica, con temas fascinantes en sus respectivas áreas de especialización.

Los bosques nublados se presentan en condiciones ambientales que varían desde semi-áridas hasta muy húmedas y se dan casos donde también son frecuentes las precipitaciones orográficas de gran intensidad y a estos puntos en particular, es que se denomina "bosques pluviales" o pluvisilva.

Dentro de nuestra reducida extensión geográfica insular, se dan ambos casos y a pesar de su importancia científica y su papel indispensable en la alimentación y el mantenimiento de los principales ríos y redes hídricas acompañantes, que garantizan la producción agrícola y pecuaria en la mayor parte del territorio de la República Dominicana y en gran medida, de las principales áreas productivas de la hermana República de Haití, todavía se desconoce la localización, ecología, composición y estructura de los mismos.

Este trabajo es la síntesis o más bien, el resumen de observaciones, apuntes y consultas que hemos realizado en todo el territorio nacional durante los último once años (1978-1989). No es un estudio detallado ni expuesto a profundidad y si alguna pretensión persigue, es la motivación a los entendidos en ciencias naturales para que incluyan a los bosques húmedos y nublados dentro de sus prioridades de investigación.

Necesitamos nuevos argumentos, nuevos elementos técnicos y una base científica para encarar el deterioro ambiental y la degradación acelerada del "patrimonio natural" de esta parte de La Española o Isla de Santo Domingo, fruto de la incomprensión y el manejo irracional de nuestros recursos naturales.

\section{Los bosques nublados y pluviales de la República Dominicana}

a) Sistema de Quita Espuela

El Sistema de Quita Espuela, en la parte oriental de la Cordillera Septentrional, está formado por una cadena de montañas alineadas en 
dirección este-oeste y que se conocen con el nombre de Firme de Quita Espuela (La Canela, Quita Espuela, Loma Vieja, El Quemado y Los Sabrosos), con una altura máxima de 985 metros sobre el nivel medio del mar.

Por su exposición directa a la acción de los vientos alisios que arrastran la humedad del anticiclón oceánico del Atlántico Norte (Trópico de Cáncer), Quita Espuela es una zona de amplia nubosidad durante todo el año y frecuentes lluvias orográficas de gran intesidad. En 1988, por ejemplo, las lluvias se mantuvieron por 8 meses seguidos y en los 4 restantes la nubosidad diurna y nocturna siempre dejó sentir sus efectos favorables sobre el régimen hídrico del área. ${ }^{\top}$

En este sistema se conjugan armoniosamente las formaciones climático - vegetales del bosque nublado y el bosque pluvial. Aunque resulta un tanto difícil notar la diferencia entre ambos, la zona del bosque pluvial apenas ocupa unos 10 kilómetros cuadrados (16,000 tareas aproximadamente) en la cima de Quita Espuela, donde las lluvias orográficas son más intensas y persistentes; mientras que la presencia del bosque nublado se extiende pr unos 700 metros hasta el firme de la vertiente norte de este sistema montañoso, cubriendo una superficie variable entre 150 y 200 kilómetros cuadrados ( 240 mil - 320 mil tareas).

En ocasiones, los cúmulos nubosos se deslizan hacia la vertiente sur de Quita Espuela; pero en realidad, el predominio del bosque nublado se mantiene en los bosques primarios y secundarios de La Canela, al norte y al este de Quita Espuela. ${ }^{2}$ En estos bosques se observan fenómenos climático- atmosféricos asociados a comunidades vegetales, que despiertan la curiosidad científica e invitan a la investigación.

En los bosques de La Canela y sus alrededores, todavía quedan mosaicos muy bien conservados de lo que fue el bosque primario. Estas muestras prístinas del bosque virgen conservan especies milenarias y varias veces centanarias como la Sabina sin Olor (Cyrilla racemiflora) con edades calculadas entre 800 y 1,200 años; Palo de Viento (Didymopanax tremulus) con más de 500 años y varios ejemplares centenarios de Nisperillo (Manilkara albescens), Capá de olor (Cordia alliodora), Cabirma (Guarea guidonia), Balatá (Manilkara bidentata), Coi (Mora abbottii), Amacey (Tetragastris balsamífera), Mara (Clophyllum calaba) y Pino de Teta (Zanthoxylum martinicense) entre otras. 
En algunos casos se observan mosaicos puros de manaclares (Prestoca montana) o asociados a los helechos machos (Helecho Arboreo) Cyathea arborea y Alsophyla sp, los que junto al Sablito (Didymopanax morototoni), son las plantas testigos o indicadoras de esta zona de vida (bosque pluvial). En cambio, el bosque nublado se caracteriza principalmente por la profusióm de epífitas (musgos, helechos, orquídeas, líquenes, bromelias y parásitas).

La nubosidad persistente del bosque nublado, mantiene un ambiente dentro de la comunidad vegetal saturado de vapor de agua, donde la humedad relativa del aire casi nunca desciende del $100 \%$. Este ambiente es ideal para el desarrollo de plantas y comunidades especiales, como es el caso de los helechos de la familia Hymenophilaceae, cuyas especies se caracterizan por la primitiva estructura de sus hojas, que no les permiten defenderse de la transpiración. ${ }^{3}$ Como representante de esta familia, en Quita Espuela se encuentra el Hymenophyllum fucoides.

Los cojines de musgos y helechos sirven de substrato a otra gran diversidad de plantas epífitas (o vegetación dependiente) donde los helechos de los géneros Elaphoglossum, Peltapteris y Grammitis; junto a las orquídeas Maxillaria coccinea y Epidendrum ramosum, son los mayores exponentes en el bosque nublado de Quita Espuela.

En este ecosistema de montaña existe un marcado endemismo a todos los niveles de organización, tanto del reino vegetal como del reino animal, muchas de cuyas especies ni siquiera se han identificado. ${ }^{2}$ No obstante, el avance de la deforestación (a causa de la agricultura y la ganaderia en áreas de exclusiva vocación forestal), está reduciendo las masas boscosas a su mínima expresión, sin que todavía conozcamos el estatus de las especies y valores biológicos únicos que ya se han descubierto.

\section{b) Diego de Ocampo}

Diego de Ocampo es la máxima elevación de la Cordillera Septentrional (1,249 metros), ubicado en su parte centro-occidental y aunque no recibe la misma cantidad e intensidad de las lluvias que se registran en Quita Espuela, también conserva una muestra singular del bosque nublado, por su exposición a los vientos alisios del norte-noreste.

El área ocupada por el bosque nublado es bastante exigua (posiblemente entre 30 y 40 kilómetros cuadrados -de 48 mil a 64 mil tareas) y se encuentra uniendo las tres montañas más altas de esta cordillera 
(Diego de Ocampo al oeste, Firme del Jamo al centro y Bocas de Licey al este) únicos puntos donde se encuentra la zona de vida bosque muy húmedo montano bajo, según el sistema de Holdridge. El piso montano bajo se encuentra a elevaciones que oscilan entre los 850 y 2,100 metros sobre el nivel del mar.

A pesar de que hay una ley (No. 5697) que protege al pico Diego de Ocampo como Zona Vedada desde 1962, su vegetación natural ha sido removida severamente por los agricultores itinerantes que desde la década del 50 se han ido ubicando en sus inmediaciones para establecer cultivos anuales y de subsistencia. También el área ha sido desmontada extensamente para establecer plantaciones permanentes de café y cacao en menor proporción.

Un año antes de la creación de la zona vedada, el Dr. José de Jesús Jiménez Almonte (principal promotor) describió y colectó muestras significativas de la flórula de esta montaña (en un viaje que realizó el 25-09-61), siendo las más comunes: ${ }^{4}$

\begin{tabular}{|c|c|c|}
\hline Nombre Científico & Nombre Común & Familia o Grupo \\
\hline Cyathea sp & Helecho Arborescente & Pteridofitas \\
\hline Elaphoglossum sp & Helecho & " \\
\hline Hymenodium crinitum & $"$ & $"$ \\
\hline Hymenophyllum hirsutum & " & $"$ \\
\hline Polidpodium aureum & " & " \\
\hline Vittaria stipitata & $"$ & " \\
\hline Lycopodium sp & " & $"$ \\
\hline Lycopodium dichotomun & $"$ & $"$ \\
\hline Lycopodium linifolium & " & " \\
\hline Lycopodium myrsimitis & " & " \\
\hline Lycopodium setaceum & $"$ & " \\
\hline Arthrostydlidium sp & Hierba & Gramíneas \\
\hline Uncinia humata & & Ciperaceas \\
\hline Euterpe globosa & Manacla & Palmaceas \\
\hline Catopsis floribunda & Bromelia & Bromeliaceas \\
\hline Tillandsia hotteana & $"$ & $"$ \\
\hline Bomarea edulis & Maya Cimarrona & $"$ \\
\hline Dichaea pendula & Orquídea & Orchidaceae \\
\hline Dichaea trichocarpa & & \\
\hline Dichaea morrissii & " & $"$ \\
\hline Elleantus capitatus & $"$ & $"$ \\
\hline Octadesmia montana & $"$ & $"$ \\
\hline
\end{tabular}




\begin{tabular}{|c|c|c|}
\hline Pleurothallis crassipes & Orquídea & Orchidaceae \\
\hline Pleurothallis velaticaulis & & \\
\hline Trichopilia fragans & " & $"$ \\
\hline Pothomarphe umbellata & Broquelejo & Piperaceas \\
\hline Cecropia peltata & Yagrumo & Moraceas \\
\hline Urera baccifera & Pringamosa & Urticaceas \\
\hline Ranunculus recurvatus & & Ranunculaceas \\
\hline Nectandra coriacea & Cigua & Lauraceas \\
\hline Cissampelos pareira & Bejuco de Ratón & Menispermaceas \\
\hline Brunellía comocladifolia & Palo de Cotorra & Brumeliaceas \\
\hline Citrus limetta & Limón Dulce & Rutaceas \\
\hline Bursera simaruba & Almácigo & Burseraceas \\
\hline Polygala penaea & Amacey & $"$ \\
\hline Ilex sp & Quicbra Hacha & Poligalaceas \\
\hline Ampelocissus robinsonii & - & Aquifoliaceas \\
\hline Passiflora sexflora & - & Passifloraceas \\
\hline Psidum guajava & Guayaba & Mirtaceas \\
\hline Ossea Lima & - & Melastomataceas \\
\hline Theophrasta jussiaei & Guayaba Cimarrona & Tcofrastaceas \\
\hline Citharesylum sp & Penda & Verbanaccas \\
\hline Solanum sp & - & Solanaceas \\
\hline Clumnea sp & Sangre de Cristo & Gensfraceas \\
\hline $\begin{array}{l}\text { Rhytidophyllum } \\
\text { aruticulatum }\end{array}$ & - & $"$ \\
\hline Siphocampylus sp & - & Lobeliaceas \\
\hline Cayaponia sp & - & Cucurbitaceas \\
\hline Anguria pedata & - & $"$ \\
\hline Penelopeia suburceolata & - & S \\
\hline Baccharis myrsinitis & Palo de Toro & Compuestas \\
\hline Euphatorium sp & - & " \\
\hline Erechthites valerianifolia & - & 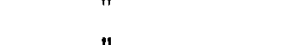 \\
\hline Gnaphalium viscosum & - & $"$ \\
\hline Liabum umbellatum & - & $"$ \\
\hline Neurolaena lobata & - & $" 1$ \\
\hline
\end{tabular}

Actualmente (septiembre 1989), justamente 28 años después del estudio florístico del Dr. Jiménez, el equipo técnico del Jardín Botánico Nacional se encuentra haciendo un reconocimiento más detallado de la flora del Diego de Ocampo. Informes preliminares nos hablan de la presencia de más de 40 especies de orquídeas y de 80 o más de helechos aparte de otras maravillas botánicas. ${ }^{5}$ 


\section{c) Zona sur-oriental de la Cordillera Central}

En el extremo sur-oriental de la Cordillera Central se dan dos manchas o pequeñas áreas de bosques nublados muy interesantes, ambas enmarcadas en el piso montano bajo y en la zona de vida del bosque muy húmedo. La primera se denomina "La Humeadora" y la scgunda, "La Valvacoa".

La Humeadora se encuentra ubicada a lo largo de la línea de divorcio de las aguas entre las cuencas de los ríos Haina y Nizao. La nubosidad persistente, a lo largo de todo el año, se aprecia perfectamente desde la Autopista Duarte, en el tramo Madrigal-La Cumbre. Las montañas más representativas son: Los Chicharrones $(1,037 \mathrm{~m}), \mathrm{El}$ Pío (1,460 m), Alto de Arroyo Grande (1,286m) y Los Calimetes (1,350 $\mathrm{m})$.

La Valvacoa, un poco más al sur de La Humeadora, se encuentra uniendo dos pequeños mosaicos del bosque muy húmedo montano bajo, entre las cuencas de los ríos Nizao y Ocoa. También, la nubosidad de esta formación es apreciable a distancia desde la Carretera Sánchez, a su paso por la Provincia Peravia (tramo Yaguate-El Número). Las dos montañas que mejor representan el bosque nublado son La Valvacoa $(1,775 \mathrm{~m})$ y La Tachuela $(1,545 \mathrm{~m})$.

La influcncia climático-hidrológica de estas dos formaciones se deja sentir significativamente en varios ríos y otros cursos menores de agua de gran importancia. La Humeadora, por ejemplo, permite apreciar su aporte al mantenimiento del régimen hídrico de los ríos Isa-Mana y Haina-Duey en cuyos cursos se hacen las dos tomas de agua principales para el acueducto de la capital dominicana.

La Humeadora tambićn alimenta los ríos Mahoma y Mahonita, que aportan directamente sus aguas a las presas de Jigüey (el primero) y Aguacate (cl segundo), y de manera indirecta, contribuyen al funcionamiento de la hidrocléctrica de Valdesia y posteriormente también beneficiarán a la ciudad de Santo Domingo, con la construcción del Acueducto -derivador Valdesia- Santo Domingo, que actualmente se encuentra en su fase final.

Pero también La Humeadora influye sobre los manantiales que dan origen (cabecera) a los ríos Maimón, Yuboa y Yuna, que además de alimentar el embalse hidroeléctrico más grande del país (Presa de 
Hatillo), juegan un papel importante insustituible en la producción agrícola y pecuaria del Bajo Yuna (Cibao Oriental).

La Valvacoa, aunque en menor proporción, también ayuda a sostener el régimen fluvial de los ríos Baní (de cuyas aguas dependen varios asentamientos humanos, tanto urbanos como rurales), Jigüey (próximo a cuya confluencia con el Río Nizao se construye la Presa Hidroeléctrica del mismo nombre) y varias corrientes menores que van a desembocar al Río Ocoa.

En ambos casos, la deforestación ha causado estragos; pero ésta ha sido más drástica en las inmediaciones de La Valvacoa en La Humeadora. Desde la llanura de Baní, se observan completamente desnudas, a roca viva, las montañas del flanco sur de La Valvacoa. En La Humeadora, aún se conservan hermosos mosaicos completamente forestados en las cabeceras de los ríos Haina-Duey e Isa-Mana.

Las especies botánicas más representativas del sistema La Humeadora son La Manacla (Prestoea montana) que se presenta formando palmares puros o mezclados con Helecho Arboreo (Cyathea sp) en las cuencas altas de los ríos Isa, Mana y Duey. Los árboles más comunes son el Yagrumo (Cecropia peltata), el Sablito (Didymopanax morototoni), Palo Ramón (Trophis domingensis), Mara (Calophyllum calaba) y Maricao (Byrsomina coriacea) entre otras.

En La Valvacoa también se dan hermosos manaclares con helechos arbóreos en los nacimientos y cabeceras de manantiales, aunque no tan extensos como en La Humeadora. Las otras especies botánicas comunes son: Amaccy (Tetragastris balsamífera) Quiebra Hacha (Krugiodendron ferreum), Caimito (Chrysophyllum oliveiforme), Penda (Cithaeroxylum fruticosum), Caya Amarilla (Mastichodendron foetidissimum), Cigua (Nectandra antillana), Cedro (Cedrela odorata) y Memiso (Muntingia calabura) entre otras.

La extensión del bosque nublado de La Humeadora alcanza unos 20 kilómetros de largo, por una anchura variable entre unos 3 y 8 kilómetros (aproximadamente $130 \mathrm{~km}^{2}$ ). En La Valvacoa en cambio, el bosque nublado cubre apenas unos 80 kilómetros cuadrados o menos.

d) Sistema de Casabito

Hemos designado como Sistema de Casabito a la porción nororiental de la Cordillera Central que va desde la cabecera del Río Camú hasta el Río Blanco e incluye a las montañas La Sal, La Golondrina, 
Firme de Casabito y la Calentura. Esta cadena montañosa, orientada en sentido norte-sur, con cierta inclinación hacia el oeste en los extremos septentrional y meridional, se opone directamente al flujo de vientos predominantes del Cibao Oriental y que a su vez es el resultado de las modificaciones locales que sufren los vientos alisios que penetran $r$ la isla por el noreste.

Este muro de contención a las corrientes de aire caliente y húmedo, representa la primera barrera natural que obliga a elevar y condensar la gran cantidad de vapor de agua proveniente del Océano Atlántico y que por las condiciones orográficas imperantes, se desplaza desde la Llanura de Nagua y la Bahía de Samaná en dirección oeste-suroeste hasta chocar con el Sistema de Casabito.

De esta manera se forma en esta área cordillerana, la mejor muestra del "bosque nublado" de la República Dominiacna, el cual comienza alrededor de los 750 metros sobre el nivel del mar (zona más baja de la Cordillera Central con este tipo de formación climático-vegetal y se eleva hacia el piso Montano Bajo, elevándose casi hasta los 1800 metros (Alto de la Calentura).

El bosque nublado del Sistema de Casabito tiene una longitud de aproximadamente 35 kilómetros y una anchura variable entre 5 y 10 kilómetros, para una superficie aproximadamente de 250 kilómetros cuadrados. La parte superior a los 800 metros de este sistema montañoso define el habitat natural de la Magnolia pallescens (Ebano Verde), la más hermosa de nuestras maderas preciosas y que sólo se encuentra en Valle Nuevo y en este punto de la Isla de Santo Domingo.

Dentro del área del bosque nublado se dan las siguientes zonas de vida según el sistema Holdridge ${ }^{6}$ :

-Bosque húmedo Subtropical (bh-S)

-Bosque muy húmedo Subtropical (bmh-S)

-Bosque muy húmedo Montano Bajo (bmh-MB)

-Bosque pluvial Subtropical (bp-S)

Dentro de las formaciones pluvial y muy húmedo montano bajo se presentan los mosaicos de palmeras (manaclares) más hermosos del país. A ambos lados de la carretera El Abanico-Constanza y a partir de los 750 metros a su paso por el Sistema de Casabito, se aprecian varias 
manchas puras de Palma Manacla (Euterpe globosa) y en otros casos, mezclada con todo tipo de helechos terrestres, arbóreos y epífitas.

La mancha del bosque pluvial Subtropical del Sistema de Casabito, es la más grande del país y cubre una superficie de aproximadamente unos 20 kilómetros cuadrados en las cuencas altas o cabeceras de los ríos Masipedro y Jima; el primero va al Yuna para contribuir o aportar sus aguas a la Presa de Hatillo y el segundo es el principal abastecedor de la Presa de Rincón.

Este sistema se encuentra actualmente seriamente amenazado por la práctica del cultivo de café a pleno sol. Varios empresarios y agrónomos promueven y provocan desmontes, incendios forestales y desbroce de los abrigos boscosos del bosque nublado y donde los helechos mantienen una cobertura completa del suelo.

La presencia de comunidades vegetales dependientes (bromelias, líquenes, orquídeas, helechos, hongos, musgos...), es algo impresionante, pero lo que más llama a la atención de los científicos, por su originalidad y escasa distribución, es la presencia de algunas plantas carnívoras. Tal es el caso de la Pingüicola casabitoana, endémica de la zona y epífita, cuando la mayoría de éstas (plantas carnívoras), tienen hábitos terrestres.

Entre las especies forestales dominantes están:

$\begin{array}{ll}\text { Ebano Verde } & \begin{array}{l}\text { Magnolia pallescens } \\ \text { Canelilla }\end{array} \\ \text { Quiebra hacha } & \begin{array}{l}\text { Krugiodendron ferreum } \\ \text { Haemianthus solicifolius }\end{array} \\ \text { Cara de Hombre } & \text { Tabebuia berterii } \\ \text { Roble cimarrón } & \text { Didymopanax tremulus } \\ \text { Palo de Viento } & \text { Brumellia comocladifolia } \\ \text { Palo de Cotorra } & \text { Trema micratha } \\ \text { Memiso } & \text { Beilsckmiedia pendula } \\ \text { Aguacatillo } & \text { Euterpe globosa } \\ \text { Manacla } & \text { Linociera caribaea } \\ \text { Tarana } & \text { Gyrotaenia myriocarpa } \\ \text { Casabito } & \end{array}$

e) Sistema de Valle Nuevo

El Sistema de Valle Nuevo, para el bosque nublado, está definido por la porción de la Cordillera Central que se extiende desde la cabecera 
del río Yuma (parte oriental) donde el Alto del Pichón es la loma más alta (2,987 msnm) hasta Loma Vieja y el Alto de Pinar Parejo (2,250 $\mathrm{msnm})$ al oeste e incluye La Chorreosa (2,672 $\mathrm{msnm})$, Alto Bandera (2,842 $\mathrm{msnm})$ y las montañas al sur del río Las Cuevas, siendo el Alto de las Avispas (2,581 msnm) la más alta.

El bosque nublado de Valle Nuevo se inicia en la zona noreste con hermosos manaclares y un bosque de latifoliadas hidrofítico que se eleva desde los 1,000 metros hasta los altiplanos de la región. En este lugar, donde se desprenden varios caños o cascadas del Río Blanco, las pendientes son tan escarpadas que su inclinación sobrepasa los $45^{\circ}$, es decir, más de un $100 \%$ y si se observa desde las montañas circunvecinas, da la impresión de que es una pared o un farallón, el obstáculos que se opone a los vientos predominantes del nordeste.

Toda la vertiente norte de los altiplanos de Valle Nuevo que va desde la cabecera del río Yuna hasta Pinar Parejo, salvo pequeños segmentos, ocupados por pinares, está cubierta por árboles de hojas anchas, principalmente por ebanales, pues es la continuación del Continuo Ecológico que constituye el habitat natural del Ebano Verde (Magnolia pallescens) que se inicia en el Sistema de Casabito.

El Ebano Verde solamente se desarrolla dentro de la formación del bosque nublado y por estas razones (principalmente), tiene requerimientos ambientales muy específicos y resulta muy limitada y localizada su distribución en La Hispaniola.

Pero el Sistema de Valle Nuevo (bosque nublado) está conformado básicamente por bosques de coníferas (más de un $85 \%$ ), donde el Pino criollo (Pinus occidentalis) es la especie forestal dominante y el Pajón Blanco (Danthonia domingensis), es la gramínea que en mayor proporción conforma el sotobosque. Ambas especies botánicas son endémicas de la Isla.

Toda la región de Valle Nuevo ocupada por el bosque nublado, muestra una gran cantidad de especies de plantas endémicas. Ya se conocen 97 plantas exclusivas de ambientes cordilleranos y una gran cantidad de epífitas (bromelias, líquenes, musgos, orquídeas, helechos...) y parásitas (Conde del Pino de flores amarillas: Dendropemon constantiae y Conde del Pino de flores rojas: Dendropemon pycnophyllus, ambas endémicas. ${ }^{7}$ 
En la cuenca alta del río Nizao se presenta la formación del bosque pluvial montano bajo (bp-MB) que apenas ocupa unos 10 kilómetros cuadrados. En el resto del área del bosque nublado se dan las zonas de vida de bosque muy húmedo Montano y Montano Bajo (bmh-M y bmh-MB) y una transición del bosque muy húmedo Montano Bajo del bosque pluvial montano bajo.

Durante los meses de diciembre, enero, febrero y a veces marzo, es común la formación de heladas o escarchas en todos los vallecitos desprovistos de vegetación superior (árboles). El bosque en este caso, es un armotiguador de las temperaturas extremas y actúa como un termostato; por estas razones durante las noches, en su interior la temperatura es más elevada que en los vallecitos (permitiendo la congelación solamente fuera del bosque); durante el día ocurre lo contrario, los vallecitos se calientan más que el bosque.

La extensión del bosque nublado del Sistema de Valle Nuevo, aunque con una forma irregular, cubre una superficie aproximada de unos 400 kilómetros cuadrados, con dimensiones que oscilan entre 30 y 35 kilómetros de largo (vertiente norte) y entre 10 y 18 kilómetros de ancho (siendo más extensa en la vertiente oriental: cuencas de los ríos Yuna, Nizao y Ocoa). La cuenta o márgenes inmediatos al curso principal del río Las Cuevas, no se incluye dentro del bosque nublado, por su gran depresión aunque las montañas a ambos lados conservan esta condición.

Las plantas típicas del Sistema de Valle Nuevo son:

Pino Criollo

Ebano verde

Podocarpus

Palo de Cotorra

Palo de Viento

Pajón Blanco

Melaito

Sarza

Palo de Reina

Senecio

Senecio

Orquídea

Orquídea

Bromelia

Helecho Arboreo

\author{
Pinus occidentalis \\ Magnolia pallescens \\ Podocarpus buchii \\ Brumellia comocladifolia \\ Didymopanax tremulus \\ Danthonia domingensis \\ Melinis minutiflora \\ Rubus domingensis \\ Lyonia tuerckheimii \\ Senecio picardae \\ Senecio constanzae \\ Lepanthopsis anthochtenium \\ Oncidium tuerckheimii \\ Catopsis floribunda \\ Cyathea sp
}




\section{f) Maciso Central}

El Maciso Central en la cordillera del mismo nombre, se extiende desde la Loma de los Camarones $(2,439 \mathrm{msnm})$ al este, hasta el Pico Nalga de Maco (1,990 msnm) al oeste, atravesando las montañas más prominentes del país: Sierra Atravesada $(2,055 \mathrm{msnm})$; La Rucilla, Duarte y La Pelona (por encima de los 3,000 msnm); Loma La Viuda (2,801 msnm); Cerro Prieto (1,849 msnm); Lomita La Piedra (2,142 $\mathrm{msnm})$ y Monte Joca (2,000 msnm). De norte a sur están las montañas Los Plásticos (2,522 msnm); Alto del Berraco (3,000 msnm); Macutico (2,561 $\mathrm{msnm})$ y Alto de la Rosa (2,322 $\mathrm{msnm})$.

Este es el sistema de bosque nublado más grande del país, cubriendo más de 1,200 kilóm tros cuadrados de superficie. Las dimensiones varían entre 120 y 155 kilómetros de largo, y entre 5 y 30 kilómnetros de ancho (9 kilómetros promedio de ancho). La máxima extensión transversal, va desde Diferencia al norte, hasta Los Fríos del sur.

El bosque nublado se encuentra en elevaciones que varían entre los 1,200 y 3,000 metros sobre el nivel del mar, en la vertiente norte de la Cordillera Central, donde predominan los vientos del nordeste; mientras que en la vertiente sur, casi nunca desciende de los 1,500 metros.

Dentro del bosque nublado de este maciso central se dan las siguientes zonas de vida, según Holdridge:

-bosque muy húmedo Montano Bajo (bmh-MB)

-bosque muy húmedo Montano Bajo de transición al bosque pluvial

Montano Bajo

-bosque pluvial Montano Bajo (bp-MB) y

-bosque muy húmedo Montano (bmh-M)

La formación del bosque pluvial Montano Bajo se presenta en dos pequeñas porciones de aproximadamente 15 kilómetros cuadrados cada una, en las lomas de Los Camarones, al sur de Manabao y Alto de la Rosa en la cuenca alta del río San Juan.

Es tal la magnitud del bosque nublado que a lo largo del año y de estaciones en estaciones, se pueden apreciar cuatro tipos de microclimas diferentes:

a) El más común o típico comportamiento del bosque húmedo, que se caracteriza por la formación de cúmulos nubosos a media mañana 
(entre las 9:00 y 11:00 a.m.) que cubren completamente en las horas de mayor insolación o radiación (1:00 p.m.) y comienza a despejarse entre las 3:00 y 4:00 p.m.

b) En períodos cortos (dos o tres días por semana) durante el año, las nubes o neblinas imperan en las primeras horas de la mañana, despejándose el ambiente alrededor de las 10:00 a.m., para volver a entordarse (presencia de nubes persistentes) al atardecer (4:00 ó 5:00 p.m.).

c) Otro micro-clima que se presenta eventualmente a lo largo del año, es la presencia de una llovizna intermitente acompañada de cúmulos nubosos durante todo el día y a veces, durante las noches.

d) Y el menos común, pero que se puede apreciar principalmente durante los meses de invierno, es la presencia de un día entero completamente despejado, aunque a lo lejos, en el horizonte, siempre se aprecien algunos cirros o cúmulos-nimbos.

Este sistema de bosque nublado cubre gran parte de los bosques mejor conservados de la Cordillera Central, principalmente de coníferas (Pino, Sabina y Podocarpus). En las zonas bajas, donde se forma el bosque nublado en la vertiente norte de la Cordillera (por debajo de los 2,000 metros) se forman prominentes bosques de latifoliadas que alcanzan su máxima expresión en las cuencas inmediatas o riberas de los ríos más importantes que descienden desde las alturas donde impera el Pino Criollo.

En la vertiente sur de la Cordillera, el bosque nublado se queda en las coníferas y salvo raras excepciones, casi nunca toca los bosques de hojas anchas. En este maciso central se encuentran los Parques Nacionales Armando Bermúdez al norte y José del Carmen Ramírez al sur.

En los múltiples vallecitos intramontanos dentro del bosque nublado, todos los años se forma escarcha o lo que es lo mismo, se producen heladas, registrándose en algunos casos temperaturas de hasta menos 12 grados centígrados (reportes de 1973), en la época invernal.

En esta zona del país, se aprecian hermosas asociaciones o combinaciones vegetales entre pinares, manaclares y helechales. Los pinares siempre están en los sitios de mejor drenaje, los helechos arbóreos en las zonas de encharque o inundables y los manaclares a lo largo de las corrientes o manantiales que como verdaderas redes hídricas descienden hacia los cursos de agua más importantes. 
La vegetación típica del bosque nublado, en esta zona de la Cordillera Central, está básicamente definida por:

Pino Criollo
Sabina
Palo de Cotorra
Manacla
Palo de Viento
Helecho arbóreo
Pajón Blanco
Lionia
Senecio
Bromelia
Tetrazygia

g) Sierra de Neiba

El bosque nublado de la Sierra de Neiba es bastante exiguo y alargado, pues solamente se presenta en las cumbres más elevadas del firme de la cadena montañosa que se inicia en el pico Media Cara $(1,842$ $\mathrm{msnm}$ ) entre Vallejuelo y Galván al este y que se desplaza hacia el oeste pasando por el Pico Neiba (2,279 msnm) hasta prácticamente la frontera con Haití.

Es decir, el bosque nublado de este sistema es un sombrero de nubes que cubre la Sierra de Neiba a partir de los 1,700 metros de altura. Aunque en algunos casos aparecen algunos pinares, la vegetación predominante es de latifoliadas asociadas con helechos arbóreos, formando abrigos boscosos muy densos y cerrados.

Casi toda la vegetación natural de la vertiente norte, donde predominan los vientos de altura que transportan el vapor de agua para forman los cúmulos nubosos, ha sido removida. Los aserraderos que funcionaron a toda capacidad hasta 1967; los cortes clandestinos que ilegalmente se hacen desde entonces y la práctica de la agricultura itinerante, ha diezmado casi por completo los bosques desde la base o pie de monte hasta aproximadamente los 1,800 metros de altitud.

Las fallas geológicas pronunciadas del Graben de Enriquillo, con pendientes que pueden alcanzar el 150\% de inclinación, han impedido todo tipo de intervención humana en la vertiente Sur, próximo al firme de la Sierra de Neiba. El bosque nublado en este caso no desciende y 
las nuibes quedan colgando como un manto de algodón tendido a lo largo de una pared.

En este caso, el bosque nublado ocupa la zona de vida del bosque muy húmedo Montano Bajo y una transición del muy húmedo Montano Bajo al bosque pluvial Montano Bajo. La extensión del bosque nublado no pasa de los 150 kilómetros cuadrados, pues aunque tiene unos 60 ó 70 kilómetros de largo, su anchura apenas varía entre los 2 y 5 kilómetros. Botánicamente, la Sierra de Neiba se puede considerar casi inexplorada, pues es muy poco lo que se conoce de su vegetación natural.

\section{h) Sierra de Bahoruco}

En la Sierra de Bahoruco se presenta un fenómeno muy interesante en la superficie ocupada por el bosque nublado. En la parte más oriental de la sierra, en la Zona de Cortico y Monteada Nueva, donde las montañas más altas apenas alcanzan los 1,600 metros sobre el nivel del mar (Loma Pie de Palo), la condición del bosque nublado se la da el vapor de agua proveniente del Mar Caribe (caso único en el país), debido a las corrientes locales de aire, que predominan durante gran parte del año, en la dirección este-oeste.

En toda esta parte predominan los bosques de latifoliadas bien desarrollados, mezclados con helechos arbóreos. Parece curioso, pero ni en la Sierra de Neiba ni en la Sierra de Bahoruco hemos observado los extensos manaclares que tipifican los bosques nublados de la Cordillera Central y la Cordillera Septentrional.

En los bosques de Monteada Nueva se están descubriendo secretos botánicos de gran valor y tomándole la palabra a los taxónomos, podemos afirmar: todavia existen maravillas biológicas por descubrir, principalmente en el reino vegetal.

La revista científica Brittonia del Jardín Botánico de Nueva York, en su número 2, volumen 11, correspondiente al año 1989, hace público el descubrimiento de una nueva especie y un nuevo género de planta para las ciencias: la Obolinga zanoni, descubierta en el mes de octubre de 1985 por Thomas Zanoni, investigador y encargado del Departamento de Botánica del Jardín Botánico Nacional "Dr. Rafael M. Moscoso".

Pero no sólo la Obolinga del bosque nublado de la Sierra de Bahoruco ha llamado la atención a la comunidad científica nacional e 
internacional, sino la gran cantidad de orquídeas, muchas también nuevas para las ciencias y descubiertas recientemente por el Dr. Donald Dodd, orquideólogo y hasta hace poco encargado del Orquideario del Jardín Botánico Nacional.

En el bosque nublado de la zona oriental de la Sierra de Bahoruco, también se encuentra la otra magnolia endémica de La Española: el Ebano Amarillo (Magnolia hamori) muy parecido, pero diferente, al Ebano Verde (Magnolia pallescens) de Casabito y Valle Nuevo.

En la parte centro-occidental de la Sierra de Bahoruco, se presenta otro fenómeno interesante y es que el bosque nublado aparece a partir de los 1,800 metros sobre el nivel del mar y donde prácticamente predomina el Pino Criollo (Pinus occidentalis) todo lo contrario a Monteada Nueva.

Aquí, el vapor de agua no parece provenir del Mar Caribe, como en el caso anterior, sino del Océano Atlántico y la humedad que se incorpora, proveniente de la evapotranspiración, a los vientos alisios de altura que se desplazan en dirección noroeste-suroeste, atravesando toda la isla de Santo Domingo.

Estos mismos vientos predominantes son los que dan lugar al bosque nublado de la Sierra de Neiba, donde el nivel de condensación parece prevalecer a partir de los 1,700 metros de altura. Aquí en la Sierra de Bahoruco (centro-oeste), el nivel de las nubes (condensación), parece prevalecer a partir de los 1,800 metros, elevándose hasta los 2,367 metros (Altos del Toro), máxima elevación de este Sistema que se prolonga hacia Haití.

Sin embargo, en estos dos bosques nublados de altura, existen diferencias fisonómicas notables, pues mientras en la Sierra de Neiba está casi ausente el Pino Criollo y abundan las latifoliadas, en esta parte de la Sierra de Bahoruco se da lo contrario, el bosque nublado se da mayormente entre pinares, aunque se presenten varios mosaicos de latifoliadas en medio de los bosques de coníferas, formados básicamente por el Palo de Viento o Tembladera (Hidymopanax tremulus). En ambos casos (Sierras de Neiba y Bahoruco), abundan las bromelias, orquídeas, líquenes y helechos entre otras formas de vida vegetales dependientes de las condiciones del bosque nublado.

En la Sierra de Bahoruco, el bosque nublado ocupa las zonas de vida del bosque muy húmedo Montano Bajo (bmh-MB), bosque muy 
húmedo Montano (bmh-M) y la transición del muy húmedo Montano Bajo al bosque pluvial Montano Bajo.

La superficie ocupada por el bosque nublado en la Sierra de Bahoruco es de aproximadamente 500 kilómetros cuadrados (unos 80 kilómetros cuadrados en la parte oriental y unos 420 en el centro-oeste). Las dimensiones van desde 110 kilómetros de longitud por 5 kilómetros promedio (de 2 a 10 kilómetros) de ancho.

\section{i) Sierra de Yamasá}

La Sierra de Yamasá es una prolongación hacia el este de la Cordillera Central y está algo separada de esta última por los valles intramontanos de Bonao y Villa Altagracia y más específicamente por cursos o microcuencas altas de los ríos Haina al sureste, Maimón al centro y Yuboa noroeste (más o menos siguiendo el tramo de la Carretera Duarte entre Villa Altagracia y Bonao).

En las cadenas de montañas que forman la Sierra de Yamasá se dan dos zonas de vida, según el Sistema Holdridge, de las más húmedas del país: a) la transición del bosque muy húmedo Subtropical con el bosque pluvial Subtropical que sólo se presenta en la Loma de los Siete Picos (cabecera de varios ríos importantes: Verde, Guanuma, Higüero y Básima); y el bosque Pluvial Subtropical (bp-S) propiamente dicho y que se da justamente en la cabecera del río Payabo.

Ambas formaciones climático-vegetales se caracterizan por la ocurrencia de fuertes precipitaciones convectivas a todo lo largo del año (con 250 días de lluvias promedio anuales). La vegetación es la misma del bosque muy húmedo Subtropical (bmh-S) que impera en toda la Sierra de Yamasá; árboles de fronda con profusión de lianas y múltiples plantas trepadoras, bromelias y orquídeas.

En las montañas más expuestas a las lluvias intensas (vertiente norte de Los Siete Picos, por ejemplo) se desarrollan mosaicos de manclares que siguen el curso principal de los ríos, arroyos y cañadas que se originan en las mismas. Bajo estas condiciones abundan la Yaya Blanca (Oxandra laurifolia), el Amacey (Tetragastris balsamífera), el Hojancho (Coccoloba pubescens), la Cabirma (Guarea guidonia) y el Copey (Clusia rosea) que junto a los Ficus sp (Higos), casi siempre se presentan como plantas estranguladoras.

En el estrato inferior, en el interior del bosque se desarrollan especies arbustivas umbrófilas típicas de estos ambientes: Pringamosa 
(Urera baccifera), Helecho arbóreo (Gyathea fulgens), Café Cimarrón o Cafecillo (Psychotria sp.) y Guayaba de Indio (Teophrasta americana) entre otras.

En la Sierra de Yamasá no se presenta el bosque nublado "per se", aunque la nubosidad sea abundante durante todo el año, debido a que las montañas son de pequeñas elevaciones sobrepasando apenas y en algunos casos los 600 metros sobre el nivel del mar.

El bosque nublado solamente se presenta cuando las formaciones orográficas no sólo se oponen a las corrientes de aire predominantes, sino cuando éstas (las montañas) sobrepasan el nivel de las nubes (nivel de condensación), obligando a éstas a chocar y cruzar entre las plantas superiores, dejando gran parte de su humedad condensada en las hojas, ramas y tallos, que luego traspasa directamente al suelo y de donde pasa a la napa freática o por la acción de la escorrentía, va a parar a los cursos de aguas superficiales (ríos, arroyos o cañadas).

\section{j) Cordillera Oriental}

La Cordillera Oriental es la misma Sierra de Yamasá, prolongada hacia el este. En la misma se presentan dos pequeñas manchas del bosque pluvial Subtropical (bp-S), al sur sureste de Miches (entre Miches y El Seibo).

Las condiciones fisonómicas de estos mismos bosque descritos para la Sierra de Yamasá son idénticas o muy similares a las que se presentan en este segmento de la Cordillera Oriental. Quizás la vegetación se vea más expuesta a la acción constante o persistente de los Alisios provenientes del Atlántico Norte, al ser éste el único muro de contención que se les opone a su desplazamiento en dirección al Ecuador.

Pero son las mismas lianas, enredaderas, helechos, bromelias, especies arbustivas del sotobosque y coronas anchas de las especies forestales anteriormente señaladas. Reiteramos, no existen diferencias fisonómicas notables, aunque la composición florística puede variar y en algunos casos, muy significativamente.

Un ejemplol de ello lo constituye el Gri-Grí (Bucida buceras) muy común en toda la Cordillera Oriental y prácticamente ausente en la Sierra de Yamasá. Algo parecido sucede con las especies Ormosia krugii (Peonía); Columnea sanguinaria (Palo Santo) y Dendropanax arboreus (Palo Malo) que también son más comunes en la Oriental que en la Sierra de Yamasá. Otras especies como la Ceiba (Ceiba 
peltandra); Guaraguao (Buchenavia capitda) y el Sablito (Didymopanax morototoni), son comunes en igual proporción en ambos casos y también son plantas típicas de áreas de alto pluviometría.

Tampoco existen bosques nublados en la Cordillera Oriental, por las mismas razones de su ausencia en la Sierra de Yamasá; montañas con elevaciones inferiores al punto de condensación, que para toda esta región del país y la isla, varía entre 680 y 800 metros de altura.

CUADRO DE SUPERFICIES DE LAS AREAS MAS HUMEDAS DEL PAIS

\begin{tabular}{|c|c|c|c|}
\hline $\begin{array}{l}\text { Ubicación } \\
\text { del Area }\end{array}$ & $\begin{array}{l}\text { Transición del Bosque } \\
\text { muy húmedo al pluvial }\end{array}$ & $\begin{array}{l}\text { Bosque } \\
\text { Pluvial }\end{array}$ & $\begin{array}{l}\text { Bosque } \\
\text { Nublado }\end{array}$ \\
\hline 1. Quita Espuela & - & $10 \mathrm{Km}^{2}$ & $200 \mathrm{~km}^{2^{*}}$ \\
\hline 2. Diego de Ocampo & - & - & $30 \mathrm{~km}^{2}$ \\
\hline 3. La Humeadora & - & - & $130 \mathrm{~km}^{2}$ \\
\hline 4. La Valvacoa & - & - & $80 \mathrm{~km}^{2}$ \\
\hline 5. Casabito & - & $20 \mathrm{~km}^{2}$ & $250 \mathrm{~km}^{2 *}$ \\
\hline 6. Valle Nuevo & $10 \mathrm{~km}^{2}$ & $10 \mathrm{~km}^{2}$ & $400 \mathrm{~km}^{2 * *}$ \\
\hline 7. Maciso Central & $11 \mathrm{~km}^{2}$ & $30 \mathrm{~km}^{2}$ & $1,200 \mathrm{~km}^{2 * *}$ \\
\hline 8. Sierra de Neiba & $8 \mathrm{~km}^{2}$ & - & $150 \mathrm{~km}^{2 * * *}$ \\
\hline 9. Sierra de Bahoruco & $10 \mathrm{~km}^{2}$ & - & $500 \mathrm{kmn}^{2 * * *}$ \\
\hline 10. Sierra de Yamasá & $11 \mathrm{~km}^{2}$ & $12 \mathrm{~km}^{2}$ & - \\
\hline 11. Cordillera Oriental & - & $18 \mathrm{~km}^{2}$ & - \\
\hline Totales & $50 \mathrm{~km}^{2}$ & $100 \mathrm{~km}^{2}$ & $2,950 \mathrm{~km}^{2}$ \\
\hline
\end{tabular}

* El Bosque Nublado incluye al Bosque Pluvial

* El Bosque Nublado incluye al Bosque Pluvial y la transición del Bosque muy húmedo hacia el Bosque Pluvial

*** El Bosque Nublado incluye a la transición del Bosque muy Húmedo hacia el Bosque Pluvial.

\section{Superficie de las áreas más húmedas del país}

Sintetizando las informaciones referentes a las áreas más húmedas del territorio nacional, observamos lo siguiente:

-En el Sistema Orográfico de la Cordillera Septentrional se presentan dos zonas ocupadas por el bosque nublado, es decir, dos áreas que 


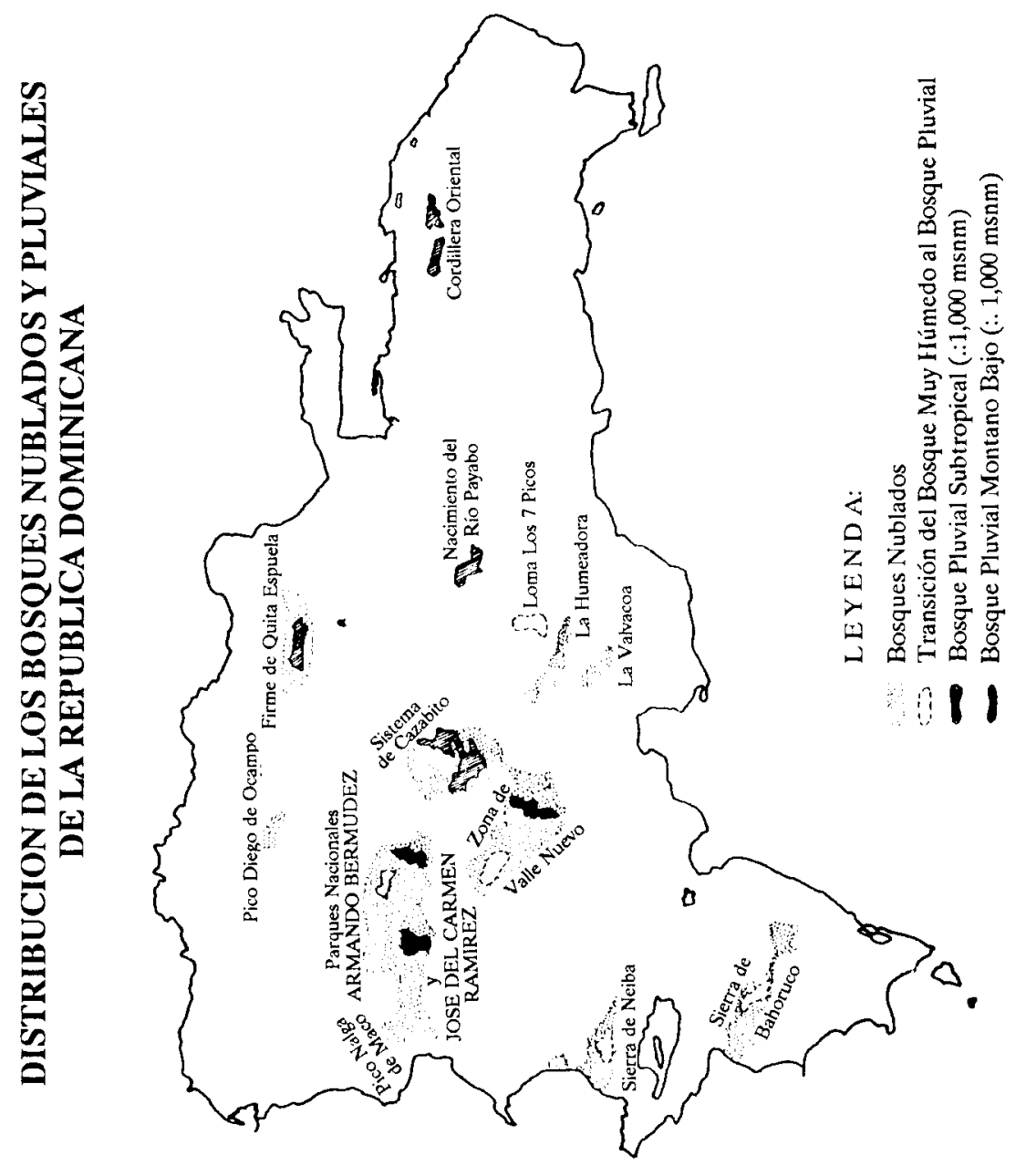


además de su alta pluviometría, reciben un aporte adicional de humedad al entrar en contacto directo las nubes y los árboles y efectuarse un intercambio energético, cuyos resultados finales siempre se expresan en un balance hídrico positivo que compresa el balance térmico negativo.

a) La primera es Quita Espuela que tiene una pequeña área del bosque pluvial subtropical $\left(10 \mathrm{~km}^{2}\right)$ que se enmarca dentro del área del bosque nublado, cuya superficie cubre unos 200 kilómetros cuadrados aproximadamente, donde nacen decenas de ríos, arroyos y cañadas.

b) La segunda área más húmeda o de incidencia del bosque nublado la constituye la zona de Diego de Ocampo (con aproximadamente 40 $\mathrm{Km}^{2}$ ), cuyas elevaciones alrededor de los 1,000 metros sobre el nivel del mar, la coloca por encima del nivel de condensación y bajo estas condiciones la vegetación superior y en especial los árboles, se convierten en atrapanubes.

-En la Cordillera Central se dan cinco zonas con incidencia del bosque nublado, la más grande e importante del país y de cuya existencia dependen en gran medida la agricultura, la generación hidroeléctrica y el abastecimiento de agua potable para los principales asentamientos humanos (incluidas las ciudades de Santo Domingo, Santiago de los Caballeros, San Juan de la Maguana, La Vega, Barahona, San Francisco de Macorís, Constanza, San Cristóbal, Baní, etc.) de la República Dominicana:

a) La primera zona de bosque nublado que podemos apreciar en el extremo oriental de la Cordillera Central es La Humeadora, cuyo aporte hídrico a los ríos del área, es indiscutible. Tiene aproximadamente unos $130 \mathrm{Km}^{2}$ de superficie.

b) La segunda zona de bosque nublado que debemos considerar es La Valvacoa, un poco más exigua que la primera (alrededor de unos 80 $\mathrm{km}^{2}$ ), pero muy cerca de la misma, con elevaciones que le permiten captar el vapor de agua que deja escapar La Humeadora en las corrientes de aire predominantes que atraviesan la isla.

c) Después tenemos el bosque nublado del Sistema de Casabito, morada natural de nuestro Ebano Verde, con algo más de 250 kilómetros cuadrados de extensión, 20 de los cuales se enmarcan además dentro de la formación bosque pluvial Subtropical.

d) En el mismo orden está la zona del bosque nublado de Valle Nuevo (con no menos de $400 \mathrm{Km}^{2}$ ) con dos pequeñas manchas o 
iormaciones climáticas especiales (una del bosque pluvial Montano Bajo, con $10 \mathrm{Km}^{2}$ y otra de igual magnitud que corresponde, según Holdridge, a la transición del bosque muy húmedo al bosque pluvial, en el piso montano bajo).

e) Por último tenemos el área más grande del bosque nublado existente en el país y que corresponde al maciso central (centrooccidental) de la Cordillera Central de la República Dominicana, cubriendo una superficie superior a los 1,200 kilómetros cuadrados, dentro de los cuales se presenta una pequeña transición del bosque muy húmedo Montano Bajo al bosque pluvial también Montano Bajo (11 $\mathrm{km}^{2}$ ) y dos porciones del bosque pluvial Motano Bajo, propiamente dicho, con $15 \mathrm{~km}^{2}$ cada una.

La importancia del régimen hídrico que se sustenta en los bosques nublados del Mosaico central y Valle Nuevo, ha sido reconocida y muy bien ponderada por casi todos los estudios e investigaciones realizadas en el pasado en esta parte del país y en especial, por los múltiples proyectos de desarrollo que se han formulado para los Valles del Cibao y San Juan (quienes constituyen la base de la producción agrícola y pecuaria nacionales).

-En la Sierra de Neiba y en las máximas elevaciones, se presenta una franja bien alargada de lo que se puede considerar el bosque nublado, con una extensión no superior a los $150 \mathrm{~km}^{2}$ y una pequeña porción incluida de $8 \mathrm{~km}^{2}$ que corresponde a la transición del bosque muy húmedo al bosque pluvial del piso montano bajo. Esta es la zona de bosque nublado más amenazada del país, pues la cobertura forestal que constituye la esencia de su existencia (en la vertiente norte), ha sido en gran medida removida y hoy permanecen en pastizales y en barbecho muy degradados.

-En la Sierra de Bahoruco, es la última región orográfica del país donde se expresa el bosque nublado en la República Dominicana y ocurre de una manera muy especial. En el extremo oriental, el bosque nublado parece recibir directamente su mayor influencia del vapor de agua proveniente del Mar Caribe, cosa que no se presenta en ninguno de los casos anteriores, mientras que los mismos argumentos o apreciaciones no se pueden hacer para el bosque nublado del resto de la Sierra, pues las formaciones nubosas que conviven con la vegetación natural, se dan a elevaciones superiores a los 1,800 metros sobre el nivel del mar. 
El bosque nubado de la Sierra de Bahoruco en su conjunto, cubre una superficie superior a los $500 \mathrm{~km}^{2}$ y solamente $10 \mathrm{~km}^{2}$ se les pueden atribuir a la formación correspondiente a la transición del bosque muy húmedo Montano Bajo al bosque pluvial de igual altitud.

-En la Cordillera Oriental, que para muchos geólogos o especialistas del área, se compone de dos mini-sistemas orográficos (Sierra de Yamasá al oeste y Sierra del Seibo al este), es el único sistema de montañas de las principales del país, que no se presenta el bosque nublado debido a sus bajas elevaciones. No obstante es una región de las más húmedas del pais, con incidencia frecuente de intensas lluvias convectivas.

a) En la Sierra de Yamasá los dos puntos más húmedos los constituyen las montañas de Los Siete Picos, con unos $11 \mathrm{~km}^{2}$ que corresponden a la transición del bosque muy húmedo al bosque pluvial de la formación subtropical, y la cabecera del Río Payabo que tiene una mancha de $12 \mathrm{~km}^{2}$ que corresponde al bosque pluvial Subtropical.

b) En la Sierra de El Seibo, es decir, en el extremo oriental de la cordillera del mismo nombre, se presentan dos pequeñas formaciones correspondientes al bosque pluvial Subtropical $\left(8\right.$ y $10 \mathrm{~km}^{2}$ de superficie respectivamente).

Resumiendo, las áreas más húmedas y frágiles (ecosistemas de montañas) de la República Dominicana cubren una superficie que se puede presentar de la siguiente manera:

-Transición del bosque muy húmedo al bosque pluvial (incluidas las formaciones Subtropical, de bajas elevaciones y Montano Bajo de elevaciones superiores) con 50 kilómetros cuadrados en su conjunto, aproximadamente un $0.1 \%$ de la superficie nacional.

-Bosque pluvial (también incluyendo las formaciones Subtropical y Montano Bajo), con 100 kilómetros cuadrados de superficie, un $0.2 \%$ del territorio nacional, $y$

-Bosque nublado, con 2,950 kilómetros cuadrados (para un $6 \%$ del territorio dominicano) y que incluye tanto las transiciones del bosque muy húmedo al bosque pluvial del piso Montano Bajoy al bosque pluvial Montano Bajo.

Si al bosque nublado, se le agregan los $41 \mathrm{~km}^{2}$ de los puntos más húmedos de la Cordillera Oriental que no entran en la superficie antes señalada para el bosque nublado $\left(11 \mathrm{~km}^{2}\right.$ de transición y 30 del pluvial 
en ambos casos "subtropical"), tenemos que la superficie de la nación dominicana con fenómenos atmosféricos especiales que se traducen en un balance hídrico muy húmedo, es de aproximadamente 3,000 kilómetros cuadrados, para un $6.2 \%$ de los $48,442 \mathrm{~km}^{2}$ que componen esta parte de la isla de Santo Domingo.

\section{Conclusiones}

Los bosques nublados, extendidos cual alfombra verde en las cimas de montañas prominentes, actúan como verdaderas esponjas para retener precipitaciones extremas y como trampa natural para la neblina y nubes impulsadas por los vientos. Estas funciones, prácticamente desconocidas 15 ó 20 años atrás, comienzan a ser valoradas y ponderadas más claramente en muchos países, principalmente en aquellos donde el agua es un factor indispensable para sus actividades productivas y de desarrollo.

Sin embargo, la deforestación (un mal universal), es el principal factor que amenaza con la desaparición de este tipo de bosques. El caso es más grave aún en aquellas naciones (tal es nuestro caso), donde muchas instituciones y órganos de decisión, todavía no tienen plena conciencia de las graves consecuencias de este flagelo que azota a los bosques tropicales.

En la República Dominicana las fronteras agrícolas están avanzando de manera acelerada hacia superficies montañosas cada vez más inclinadas y lluviosas sin que esta tendencia sea detenida o revertida por los poderes públicos cuya misión, precisamente, debe ser salvaguardar y conservar áreas con características únicas e insustituibles y que como los bosques nublados, pierden todas sus funciones al removérsele su cubierta vegetal superior.

Las autoridades, profesionales y técnicos no sólo se muestran impotentes ante la desaparición acelerada de la floresta nativa, sino que se han unido a una corriente desarrollista que vela por el "manejo sostenido" que se orienta hacia la utilización de los bosques de tal forma que no se agote el potencial productivo de los mismos.

Pero sucede que los bosques nublados no se prestan para el manejo sostenido debido a sus condiciones de fragilidad y delicadeza intrínsecas:

1. Las condiciones climáticas (especialmente la alta humedad persistente y las precipitaciones frecuentes) son totalmente desfavorables 
a las actividades silvícolas (corte, extracción, levantes y transporte de madera), no importa su modalidad o género.

2. Las condiciones topográficas (pendientes pronunciadas y relieve en extremo irregular) son más desfavorables aún, máxime si se toma en cuenta o se le suma la humedad de los suelos.

3. De igual manera, lo mismo podríamos decir de las condiciones edáficas, pues además de favorecerle la inclinación, los suelos son muy propensos a la erosión a causa de la escorrentía, la erodabilidad de la roca madre e inestabilidad del piso forestal cuando se remueve total o parcialmente la capa vegetal.

4. La función hidrológica de los bosques nublados es insustituible y ni la madera, ni la producción agrícola o pecuaria pueden compensar el costo de su pérdida.

5. Pero la función ecológica, aunque no se valore en términos ecológicos o de desarrollo, es de las más importantes, por la protección de comunidades de flora y fauna endémicas y la conservación de recursos genéticos.

6. $\mathrm{Y}$ hay quienes agregan a las funciones del bosque nublado, la protección de elementos paisajísticos particulares, como son la majestuosidad de los árboles de fronda, adornados de epífitas y el entorno envuelto en nubes que se deshacen o mueren lentamente, sin que lleguen a agotarse por completo.

7. Además, la función de museo viviente y laboratorio biológico, equipado por miles o millones de años y sometidos al juego de los procesos evolutivos; tampoco puede ser pasada por alto.

Para el equipo de investigadores de las Naciones Unidas que realizó el estudio Los Bosques Nublados en el Trópico Húmedo:

iniciar, apoyar y promover acciones para la protección de los bosques nublados tropicales es una tarea importante para el futuro. Se deben reconocer no sólo sus valores ecológicos y su importancia como ecosistema tan especial, sino también su papel hidrológico como reguladores de agua en las cuencas hidrográficas en combinación con su capacidad de conservar los suelos en las laderas de montañas. 9

En nuestra opinión, es necsario hacer llegar estas ideas (que por nuevas e incompletas que sean, no dejan de ser indispensables para orientar las estrategias de conservación de los bosques nublados), a la comunidad científica nacional, a los especialistas, a las autoridades y 
decisores y a todo aquel que se dedique o se interese en la protección del ambiente y la naturaleza dominicana.

\section{LITERATURA CITADA}

1. Almánzar, V. Reconocimento ecológico de la Selva Nublada Quita Espuela. Tesis de grado. Universidad Nordestana. San Francisco de Macorís, R. D. 1988.

2. S.E.A. Situación actual de los recursos naturales en la Loma Quita Espuela. Propuesta para su manejo integrado. Secretaría de Estado de Agricultura-Servicio Alemán de Cooperación Técnica Social. Divulgación Técnica S.E.A. Santo Domingo, R. D. 1988.

3. Haager, Johannes. Apuntes de Cátedras, Ecología Vegetal. City College. Universidad Autónoma de Santo Domingo, Santo Domingo, R. D. 1989.

4. Jiménez, J. Colectas de plantas/Flórula Diego de Ocampo. Boletín Actualidad Ecológica, No. 4, Año 3 (Agosto - Septiembre), 1981. Sociedad Ecológica del Cibao. Santiago de los Caballeros, R. D. 1961.

5. Abramo, Domeneca. Comunicación personal, 1989.

6. Holdridge, L. Ecología basada en zonas de vida. IICA. San José, Costa Rica. 1982.

7. Marcano, Eugenio de Js. Comunicación personal, 1989.

8. Barneby, R. Obolinga, A new genus of Mimosaceae Tribe Ingeae From Hispaniola. Brittonia, 41(2). New York Botanical Garden. Bronx, N.Y. 10458-1526. USA. 1989.

9. Stadtmuller, T. Los Bosques Nublados en el Trópico Húmedo. Universidad de las Naciones Unidas. Centro Agronómico Tropical de Investigación y Enseñanza. Turrialba, Costa Rica. 1987. 